\title{
Oral prodrug of remdesivir parent GS-441524 is efficacious against SARS-CoV-2 and a variant of concern in ferrets
}

\section{Richard Plemper ( $\square$ rplemper@gsu.edu )}

Georgia State University https://orcid.org/0000-0003-2034-2107

\section{Robert Cox}

Georgia State University https://orcid.org/0000-0003-0620-1674

Josef Wolf

Georgia State University

Carolin Lieber

Georgia State University

Julien Sourimant

Georgia State University https://orcid.org/0000-0002-1660-1666

\section{Michelle Lin}

University of Washington https://orcid.org/0000-0002-6925-9948

\section{Darius Babusis}

Gilead Sciences (United States) https://orcid.org/0000-0001-5599-1671

\section{Venice Du Pont}

Gilead Sciences Inc.

Julie Chan

Gilead Sciences Inc.

\section{Kim Barrett}

Gilead Sciences Inc.

\section{Rao Kalla}

Gilead Sciences Inc.

\section{Kwon Chun}

Gilead Sciences, Inc.

\section{Richard Mackman}

Gilead Sciences, Inc.

\section{Chengjin Ye}

Texas Biomedical Research Institute

\section{Tomas Cihlar}

Gilead Sciences, Inc. 
Texas Biomedical Research Institute https://orcid.org/0000-0001-7084-0804

\section{Alexander Greninger}

University of Washington

John Bilello

Gilead https://orcid.org/0000-0003-4327-1727

\section{Biological Sciences - Article}

Keywords: Oral Bioavailability, Variants of Concern, Relevant Animal Model, Virus Replication

Posted Date: July 30th, 2021

DOl: https://doi.org/10.21203/rs.3.rs-662905/v1

License: (9) This work is licensed under a Creative Commons Attribution 4.0 International License. Read Full License

Version of Record: A version of this preprint was published at Nature Communications on November 5th, 2021. See the published version at https://doi.org/10.1038/s41467-021-26760-4. 
1 Oral prodrug of remdesivir parent GS-441524 is efficacious against SARS-CoV-2 and a variant of concern in ferrets

3 Robert M. Cox ${ }^{1}$, Josef D. Wolf ${ }^{1}$, Carolin M. Lieber ${ }^{1}$, Julien Sourimant ${ }^{1}$, Michelle J Lin ${ }^{2}$, Darius

4 Babusis $^{3}$, Venice Du Pont ${ }^{3}$, Julie Chan ${ }^{3}$, Kim Barrett $^{3}$, Rao Kalla ${ }^{3}$, Kwon Chun ${ }^{3}$, Richard L. Mackman ${ }^{3}$,

5 Chengjin $\mathrm{Ye}^{4}$, Tomas Cihlar ${ }^{3}$, Luis Martinez-Sobrido ${ }^{4}$, Alexander L. Greninger ${ }^{2}$, John P. Bilello ${ }^{3}$,

6 Richard K. Plemper ${ }^{1 *}$

7

$8 \quad{ }^{1}$ Center for Translational Antiviral Research, Institute for Biomedical Sciences, Georgia State

9 University, Atlanta, GA, USA

$10{ }^{2}$ Virology Division, Department of Laboratory Medicine and Pathology, University of Washington,

11 Seattle, WA

$12{ }^{3}$ Gilead Sciences Inc., Foster City, CA, USA

$13{ }^{4}$ Texas Biomedical Research Institute, San Antonio, TX, USA.

14

15

16

17

18

19 Short title: Oral remdesivir analog blocks SARS-CoV-2

20

21

$22 *$ to whom correspondence should be addressed: rplemper@gsu.edu 


\section{Abstract}

Remdesivir is the only small-molecule antiviral approved to date for COVID-19 treatment, but its

26 wider use is limited by intravenous delivery. An orally bioavailable remdesivir analog may boost

27 therapeutic benefit by facilitating early administration to non-hospitalized patients. This study

28 characterized the anti-SARS-CoV-2 efficacy of GS-621763, an oral prodrug of remdesivir parent

29 nucleoside GS-441524. Both GS-621763 and GS-441524 inhibited SARS-CoV-2, including variants of

30 concern (VoC) in cell culture. Oral GS-621763 was efficiently converted to plasma metabolite GS-

31441524 , and in lungs to the triphosphate metabolite identical to that generated by remdesivir,

32 demonstrating a consistent mechanism of activity. Twice-daily oral administration of $10 \mathrm{mg} / \mathrm{kg}$ GS-

33621763 reduced SARS-CoV-2 burden to near-undetectable levels. When dosed therapeutically against

34 VoC P.1 gamma $(\gamma)$, oral GS-621763 blocked virus replication and prevented transmission to untreated

35 contact animals. These results demonstrate therapeutic efficacy of a much-needed orally bioavailable

36 analog of remdesivir in a relevant animal model of SARS-CoV-2 infection. 
Introduction

The global COVID-19 pandemic has resulted in far more than 150 million cases and an estimated 3.5 million deaths. The socioeconomic and geopolitical impact of the pandemic is unprecedented and will most likely become fully evident only in post-pandemic years. To date, the ribonucleoside analog remdesivir is the only small molecule antiviral therapeutic approved by the FDA for the treatment of COVID-19 ${ }^{1}$. Originally discovered for respiratory syncytial virus ${ }^{2}$, remdesivir is a monophosphoramidate prodrug that must be administered intravenously ${ }^{3}$. The obligatory parenteral delivery

44 limits access of non-hospitalized COVID-19 patients to remdesivir treatment in early disease stages, thereby significantly narrowing the therapeutic window in which a direct-acting antiviral targeting an acute viral infection is expected to have its greatest effect ${ }^{4}$.

Orally active COVID-19 therapeutics have potential to maximize patient benefit and ideally prevent progression to severe disease. Several oral therapeutic candidates such as EIDD-2801/molnupiravir ${ }^{5-7}$ and $\mathrm{AT}-527^{8}$ are in advanced stages of clinical trials, but currently only remdesivir has shown confirmed efficacy for COVID-19 treatment in humans ${ }^{9}$. Following intravenous administration and despite its short

51 plasma half-life, remdesivir is sufficiently stable in non-rodent species to distribute to tissues such as the 52 lung, where it is rapidly converted to its monophosphate metabolite and then efficiently anabolized to 53 the bioactive triphosphate GS-443902 (Fig. 1a) ${ }^{2}$. An alternative strategy to generate GS-443902 could 54 be to directly administer the remdesivir parent nucleoside GS-441524, but due to less efficient metabolism of GS-441524 to its monophosphate, higher daily systemic exposures of GS-441524 than those obtained from intravenous remdesivir are required to generate equivalent concentrations of GS443902 in lung tissue. In order to obtain high exposures after oral delivery, a prodrug strategy to improve oral absorption was employed leading to the identification of GS-621763, which demonstrated high oral bioavailability in several species ${ }^{2}$. 
SARS-CoV-2 efficiently infects mustelids such as ferrets and mink, and both direct and reverse

61 zoonotic transmission between mink and humans have been reported ${ }^{10,11}$. Infected ferrets show only

62 mild clinical signs, but the virus readily replicates in the upper respiratory tract and shed virus load in

63 nasal lavages is high, supporting efficient animal-to-animal transmission ${ }^{6}$. Consequently, ferrets

64 recapitulate the presentation of SARS-CoV-2 in the majority of human cases, especially in children and 65 younger adults ${ }^{12,13}$.

66 In this study, we used the ferret model to test oral anti-SARS-CoV-2 efficacy of GS-621763. The

67 GS-621763 prodrug is pre-systemically hydrolyzed to afford high systemic exposures of GS-441524

68 (Fig. 1a). Having determined the plasma pharmacokinetic (PK) profile of GS-621763 in ferrets, we

69 examined the effect of oral GS-621763 administered therapeutically against the original SARS-CoV-2

70 USA-WA1/2020 (WA1/2020) strain and the recently emerged, highly prevalent VoC P.1 $(\gamma)$ lineage ${ }^{14,15}$.

\section{Results}

Prior to in vivo testing, we assessed antiviral potency of both GS-621763 and its metabolite GS441524 against lineage A isolate WA1/2020 and three recently emerged VoC, hCoV-

19/USA/CA_UCSD_5574/2020 (( $\alpha)$, lineage B.1.1.7; CA/2020), hCoV-19-South Africa/KRISP-

K005325/2020 (( $\beta)$, lineage B.1.351; SA/2020), and hCoV-19/Japan/TY7-503/2021 (( $\gamma)$, lineage P.1;

$\mathrm{BZ} / 2021)$ in cultured cells.

\section{Antiviral Activity against VoCs}

Half-maximal effective concentrations $\left(\mathrm{EC}_{50}\right)$ were highly consistent, ranging from 0.11 to $0.73 \mu \mathrm{M}$

80 for GS-621763 (Fig. 1b, extended data Table 1) and from 0.11 to $0.68 \mu \mathrm{M}$ for GS-441524 (Fig. 1c,

81 extended data Table 1). Analogous potency ranges were obtained when luciferase expressing WA1/2020

82 reporter viruses were examined in dose-response assays in A549 cells stably expressing human ACE2 
83 (A549-hACE2) (extended data Table 1). Toxicity-testing of GS-621763, remdesivir, and GS-441524 in different cell lines and primary human cells derived from different donors revealed half-maximal cytotoxic concentrations $\left(\mathrm{CC}_{50}\right)$ of 40 to $>100 \mu \mathrm{M}$ (Fig. 1d, extended data Table 1), 36 to $>100 \mu \mathrm{M}$ (extended data Fig. 1, extended data Table 1) and >100 $\mu \mathrm{M}$ (Fig. 1e, extended data Table 1), respectively, corresponding to selectivity indices $\left(\mathrm{SI}=\mathrm{CC}_{50} / \mathrm{EC}_{50}\right)$ of $\mathrm{GS}-621763>137$ in VeroE6 and $88>51$ in A549-ACE2 cells.

Assessment of GS-621763 plasma PK parameters in the ferret revealed excellent oral bioavailability

91 (Fig. 2a), extensive cleavage pre-systemically to generate high exposures of GS-441524 in the blood

92 (extended data Table 2), efficient distribution to soft tissues of the respiratory system (lung), and confirmed anabolism to bioactive GS-443902 (extended data Table 3). Following a single $30 \mathrm{mg} / \mathrm{kg}$ oral dose of GS-621763 in ferrets, the daily systemic exposure (AUC0-24h) of GS-441524 was $81 \mu \mathrm{M} . \mathrm{h}, 4.5$ fold higher than the exposure following IV remdesivir at $10 \mathrm{mg} / \mathrm{kg}$ and approximately 12 -fold greater than that observed following an $200 \mathrm{mg}$ IV remdesivir dose in human ${ }^{16}$. Lower levels of bioactive GS443902 were formed from oral $30 \mathrm{mg} / \mathrm{kg}$ GS-621763 dosing compared to $10 \mathrm{mg} / \mathrm{kg}$ IV remdesivir (extended data Table 3), illustrating the difference in intracellular activation efficiency of the phosphoramidate prodrug remdesivir compared to systemic parent nucleoside GS-441524.

\section{Prophylactic efficacy in ferrets}

To test antiviral efficacy, we infected ferrets intranasally with $1 \times 10^{5}$ plaque forming units (pfu) of

102 WA1/2020, followed by twice daily (b.i.d.) oral treatment with GS-621763 at $20 \mathrm{mg} / \mathrm{kg}$ body weight for 103 four days (Fig. 2b). Treatment was initiated at the time of infection, nasal lavages collected in 12-hour 104 intervals, and respiratory tissues harvested 4 days after infection. Shed SARS-CoV-2 load in nasal 105 lavages of vehicle-treated animals reached plateau 1.5 days after infection at approximately $1 \times 10^{4}$ 
$106 \mathrm{pfu} / \mathrm{mL}$, whereas virus was transiently detectable in lavages of only one ferret of the GS-621763-

107 treatment group at 12 hours after infection (Fig. 2c). Clinical signs overall are minor in the ferret model ${ }^{6}$

108 However, only animals of the vehicle group showed elevated body temperature (Fig. 2d) and reduced

109 weight gain (Fig. 2e). Virus was undetectable in the nasal turbinates extracted from treated animals 4

110 days after infection, compared to a robust load of approximately $5 \times 10^{4} \mathrm{pfu} / \mathrm{g}$ nasal turbinate of animals

111 of the vehicle group (Fig. 2f). Viral RNA copy numbers found in lavages (Fig. 2g) and turbinates (Fig.

112 2h) mirrored the infectious titer results, revealing a consistent, statistically significant difference

113 between vehicle and treatment groups of two and three orders of magnitude, respectively. Consistent

114 with prior studies ${ }^{6}$, no infectious virions or viral RNA were detectable in the lower respiratory tract (Fig. $1152 \mathrm{i}, \mathrm{j})$.

116 Therapeutic efficacy and lowest efficacious dose

117 To determine the lowest efficacious dose in a clinically more relevant therapeutic setting, we

118 initiated oral treatment 12 hours after infection at the $10 \mathrm{mg} / \mathrm{kg}$ and $3 \mathrm{mg} / \mathrm{kg}$ body weight levels,

119 administered b.i.d. (Fig 3a). EIDD-2801/molnupiravir at $5 \mathrm{mg} / \mathrm{kg}$ b.i.d. was given as reference following

120 an identical therapeutic b.i.d. regimen ${ }^{6}$. Shed virus load was significantly lower in all treated animals

121 than in the vehicle group within 12 hours of treatment onset (Fig. 3b). Virus load in nasal lavages of

122 ferrets receiving GS-621763 at $3 \mathrm{mg} / \mathrm{kg}$ plateaued approximately one order of magnitude lower than in 123 those from vehicle animals, while treatment with GS-621763 at $10 \mathrm{mg} / \mathrm{kg}$ or EIDD-2801/molnupiravir

124 reduced shedding to near-detection level by day 3 after infection. Consistent with this inhibitory effect, 125 treatment with $3 \mathrm{mg} / \mathrm{kg} \mathrm{GS}-621763$ reduced burden in the turbinates by one order of magnitude (Fig.

$1263 \mathrm{c})$, while virus burden approached the limit of detection in animals of the $10 \mathrm{mg} / \mathrm{kg} \mathrm{GS}-621763$ and

127 EIDD-2801/molnupiravir treatment groups. No significant differences in clinical signs were noted 128 between vehicle animals and any of the treatment groups (Fig. 3d,e). 
Viral RNA was detectable in nasal lavages and turbinates of all animals, underscoring efficient

130 infection. However, RNA copies showed a statistically significant mean reduction in the $10 \mathrm{mg} / \mathrm{kg} \mathrm{GS}$ -

131621763 and EIDD-2801/molnupiravir groups compared to vehicle (Fig. 3f,g). These results confirm oral 132 efficacy of therapeutic GS-621763 against WA1/2020 in a relevant animal model of upper respiratory 133 infection.

134 Inhibition of replication and transmission of a the $\mathrm{BZ} / 2021 \mathrm{VoC}$

135 To probe the anti-SARS-CoV-2 indication spectrum of GS-621763, we applied the efficacious 136 regimen, $10 \mathrm{mg} / \mathrm{kg}$ GS-621763 b.i.d. started 12 hours after infection, to recently emerged VoC BZ/2021 13717 in a combined efficacy and transmission study (Fig. 4a). After an initial replication delay, shed virus 138 became detectable in vehicle-treated animals 1.5 days after infection, then rapidly reached a robust 139 plateau of nearly $10^{4} \mathrm{pfu} / \mathrm{mL}$ nasal lavage on day 2 after infection (Fig. 4b). Quantitation of viral RNA 140 copies mimicked the profile of the infectious titers, although a low viral RNA load was present in 141 lavages already on the first day after infection (Fig. 4c). Viral titers and RNA copies in nasal turbinates 142 determined 4 days after infection were likewise high, ranging from $10^{4}$ to $10^{5} \mathrm{pfu} / \mathrm{g}$ tissue (Fig. $\left.4 \mathrm{~d}\right)$ and $14310^{8}$ to $10^{10}$ RNA copies/g tissue (Fig. 4e), respectively. However, no infectious BZ/2021 virions or viral 144 RNA were detected in the lungs of any of these animals (Fig. 4f,g), and no clinical signs such as changes 145 in body weight or fever emerged (extended data Fig. 2a,b). This presentation mimicked our previous 146 experience with WA1/2020 ${ }^{6}$, indicating that BZ/2021 does not invade the ferret host more aggressively 147 than WA1/2020. Treatment of BZ/2021 infection with oral GS-621763 was highly efficacious, reducing 148 both shed virus burden and tissue titers to undetectable levels (Fig. 4b,d) and lowering viral RNA copies 149 in nasal lavages and turbinates by over three orders of magnitude (Fig. 4c,e).

150 Whole genome sequencing of the virus inoculum and virus populations extracted from nasal 151 turbinates confirmed the presence of mutations characteristic for the P.1 VoC ${ }^{18}$ (Fig. 4h, supplementary 
152 dataset 1). In addition, we noted an L260F substitution in nsp6 associated with SARS-CoV-2 adaptation

153 to weasels ${ }^{10}$ that had a $60 \%$-allele frequency in the BZ/2021 inoculum. Four days after infection of

154 ferrets, this mutation had become fully dominant and a second characteristic weasel mutation, Y453F in 155 the spike protein that was first noted in several clusters of SARS-CoV-2 outbreaks in mink farms ${ }^{10}$, had 156 emerged in addition (Fig. 4h, supplementary dataset 1). We furthermore noted the presence of an F184V 157 exchange in nsp6 of the BZ/2021 inoculum, which arose during amplification in VeroE6 cells ${ }^{18}$ and was 158 rapidly counterselected against in the ferret host. In contrast, the WA1/2020 inoculum used for our ferret 159 studies did not contain any unreported additional changes (Fig. 4h, supplementary dataset 2). WA1/2020 160 also acquired a weasel-characteristic mutation when passaged through ferrets, N501T in the receptor 161 binding domain of the spike protein ${ }^{10}$, but no changes in nsp6 were detected and only one animal 162 harbored a virus population with Y453F substitution in low (8\%) allele frequency. Neither the GS163 621763-experienced BZ/20201 nor WA1/2020 populations contained remdesivir resistance mutations 164 previously selected in SARS-CoV-2 (i.e. E802D in nsp12 ${ }^{19}$ ) or the related mouse hepatitis virus ${ }^{20}$ (i.e. 165 F476 L and V553L in nsp12) nor did any new variants arise in nsp12 at $>5 \%$ allel frequency, when 166 viruses were extracted from treated animals at the time of termination (Fig. 4h, supplementary datasets $1671,2)$.

168 All vehicle-treated animals efficiently transmitted VoC BZ/2021 to untreated direct contact ferrets 169 (Fig. 4b-e). Co-housing was started 54 hours after infection and continued until termination of the 170 source animals. Shed BZ/2021 replicated in the contacts without delay, becoming first detectable in 171 nasal lavages within 12 hours after initiation of co-housing. This altered replication profile corroborated $172 \mathrm{BZ} / 2021$ adaptation to the ferret host in the source animals, and virus populations recovered from 173 contacts of vehicle-treated source animals indeed contained both the L260F exchange in nsp6 and the 174 Y453F mutation in spike (Fig. 4h, supplementary dataset 1). Consistent with efficient inhibition of 
$175 \mathrm{BZ} / 2021$ replication in the treated source animals by oral GS-621763, treatment completely blocked

176 virus transmission to untreated direct-contact animals. None of the contacts of treated source ferrets shed

177 infectious particles or viral RNA at any time (Fig. 4b,c), infectious viral particles were absent from nasal

178 turbinates 5.5 days after initiation of co-housing (Fig. 4d), and only a low level of viral RNA $\left(<10^{5}\right.$

179 copies/g nasal turbinate) was detected in nasal turbinates of the contact animals (Fig. 4e).

180

181 Discussion

182 This study demonstrates oral efficacy of the remdesivir analog GS-621763 against the early clinical 183 SARS-CoV-2 isolate WA1/2020 and one of the recently emerged VoC in the ferret model. After oral 184 administration of GS-621763, only low systemic levels of intact prodrug were transiently observed. The 185 parent nucleoside GS-441524 was the major metabolite detected in blood, more than three orders of 186 magnitude higher than intact prodrug, indicating efficient conversion upon intestinal absorption. GS187441524 metabolism inside cells to the same intracellular bioactive triphosphate GS-443902 in tissues 188 links GS-621763 to the well-understood mechanism of action of remdesivir, which blocks SARS-CoV-2 189 replication by triggering delayed chain termination of the nascent viral RNA chain ${ }^{21}$ and/or template190 dependent inhibition after incorporation into viral antigenomic RNA ${ }^{22}$. Systemic GS-441524 is less 191 efficient at forming the bioactive GS-443902 in the lungs of ferrets compared to systemic remdesivir, 192 but the exposure of GS-441524 achieved following oral dosing of GS-621763 is sufficient to overcome 193 this difference and generate substantial levels of bioactive GS-443902. The more persistent but lower 194 plasma levels of GS-441524 following intravenous remdesivir are a result of efficient formation of 195 triphosphate in tissues from remdesivir, followed by slow dephosphorylation to GS-441524 which then 196 appears in plasma, as evidenced by its similar half-life with that of the triphosphate GS-443902 in $197 \mathrm{PBMC}^{16}$. 
Although GS-443902 levels in ferret respiratory tissues after a single $30 \mathrm{mg} / \mathrm{kg}$ oral dose of GS-

621763 trailed those of intravenous $10 \mathrm{mg} / \mathrm{kg}$ remdesivir by approximately 4-fold at 24 hours, a minimal

daily oral dose of $20 \mathrm{mg} / \mathrm{kg} \mathrm{GS}-621763$ (10 mg/kg b.i.d.) provided full therapeutic benefit, rapidly

201 reducing shed virus titers to near-undetectable. As the GS-443902 active metabolite is quantitated from

202 gross tissue samples, we cannot compare GS-443902 levels resulting from either GS-621763 or

203 remdesivir dosing in distinct respiratory cell types. If human oral bioavailability of GS-621763 is

204 consistent with that observed in ferrets, and the model is predictive of antiviral potential, the dose levels

205 identified in the ferret study as fully efficacious in upper respiratory tract correspond to a feasible human 206 daily dose of approximately $250 \mathrm{mg}$ oral GS-621763.

207 An observed difference of approximately two to three orders of magnitude between infectious titers 208 and viral RNA copy numbers in the ferrets closely resembled that described in earlier reports ${ }^{6,23,24}$, 209 demonstrating high reproducibility of the model. At present, the correlation between upper respiratory 210 tract SARS-CoV-2 load at early stages after human infection and the likelihood of progression to viral 211 pneumonia and severe COVID-19 has not been fully understood. However, clinical studies have linked 212 higher upper respiratory virus burden to a heightened patient risk of developing severe COVID-19, 213 requiring intubation or intensive care, and having an unfavorable outcome ${ }^{25}$. Based on these clinical data 214 and the rapid reduction of upper respiratory virus burden seen in the ferret model, we expect that early 215 initiation of oral treatment, ideally immediately following a positive diagnostic test and before the onset 216 of clinical signs ${ }^{26,27}$, holds high promise to block viral invasion of the lower respiratory tract and viral 217 pneumonia that necessitates hospitalization.

218 Recently emerged SARS-CoV-2 V such as BZ/2021 are a major concern because of their high 219 prevalence and increased frequency of transmission ${ }^{14,28}$. Ferrets infected with BZ/2021 did not develop 220 more severe clinical signs than after infection with WA1/2020 and the virus did not advance to the lower 
221 respiratory tract, indicating that pathogenicity of this $\mathrm{VoC}$ is not fundamentally different from that of 222 WA1/2020 in this model. Peak viral burden and shed virus loads of BZ/2021-infected ferrets were 223 equivalent to those of WA1/2020. Reflecting that analysis of circulating SARS-CoV-2 VoC worldwide 224 has yielded no evidence of widespread transmission of remdesivir-resistant strains ${ }^{19,20,29}$, GS-621763 225 and its metabolite GS-441524 were comparably potent against CA/2020, SA/2020, and BZ/2021 as 226 against WA1/2020 in cell culture. Importantly, oral GS-621763 was highly efficacious against BZ/2021 227 in vivo, reducing infectious titers to near-undetectable levels rapidly after treatment initiation. Whole 228 genome sequencing of GS-621763-experienced viruses recovered from treated ferrets likewise revealed 229 no allele variations in candidate remdesivir resistance sites, indicating that SARS-CoV-2 does not 230 rapidly escape from inhibition by GS-621763 in vivo.

231 Based on the high transmissibility of BZ/2021 in the field and very efficient direct contact 232 transmission of SARS-CoV-2 between ferrets ${ }^{30}$, we assessed the impact of oral GS-621763 on BZ/2021 233 spread among ferrets. To recapitulate what we consider a realistic scenario for exploring 234 pharmacological interference in community transmission, we therapeutically treated the infected source 235 animals but left their contacts untreated. In this relevant experimental setting, treatment with the same 236 GS-621763 dose of $10 \mathrm{mg} / \mathrm{kg}$ b.i.d. completely blocked transmission of BZ/2021, resembling the 237 performance of oral EIDD-2801/molnupiravir in the model ${ }^{6}$.

238 These results build confidence that a new generation of orally available broad-spectrum antivirals is 239 emerging that should allow initiation of treatment early after infection and promise to efficiently 240 interrupt community transmission chains. In addition to immediately contributing to ending the COVID24119 pandemic and containing the continued evolution of increasingly contagious SARS-CoV-2 VoC ${ }^{31-33}$, 242 an oral analog of the broad-spectrum antiviral drug remdesivir with confirmed in vivo efficacy such as 243 GS-621763 may become a cornerstone in the first-line defense against future pandemic threats. 


\section{Main References}

2461 U.S. Food \& Drug Administration. FDA Approves First Treatment for COVID-19. https://www.fda.gov/news-events/press-announcements/fda-approves-first-treatment-covid-19 (retrieved on 06/06/2021).

2 Mackman, R. L. et al. Prodrugs of a 1'-CN-4-Aza-7,9-dideazaadenosine C-Nucleoside Leading to the Discovery of Remdesivir (GS-5734) as a Potent Inhibitor of Respiratory Syncytial Virus with Efficacy in the African Green Monkey Model of RSV. J. Med. Chem. 64, 5001-5017, doi:10.1021/acs.jmedchem.1c00071 (2021).

3 Siegel, D. et al. Discovery and Synthesis of a Phosphoramidate Prodrug of a Pyrrolo[2,1f][triazin-4-amino] Adenine C-Nucleoside (GS-5734) for the Treatment of Ebola and Emerging Viruses. J. Med. Chem. 60, 1648-1661, doi:10.1021/acs.jmedchem.6b01594 (2017).

4 Meganck, R. M. \& Baric, R. S. Developing therapeutic approaches for twenty-first-century emerging infectious viral diseases. Nat. Med. 27, 401-410, doi:10.1038/s41591-021-01282-0 (2021).

5 Toots, M. et al. Characterization of orally efficacious influenza drug with high resistance barrier in ferrets and human airway epithelia. Sci. Transl. Med. 11, doi:10.1126/scitranslmed.aax5866 (2019).

6 Cox, R. M., Wolf, J. D. \& Plemper, R. K. Therapeutically administered ribonucleoside analogue MK-4482/EIDD-2801 blocks SARS-CoV-2 transmission in ferrets. Nat Microbiol 6, 11-18, doi:10.1038/s41564-020-00835-2 (2021).

7 Wendy P. Painter, W. H., Jim A. Bush, Firas Almazedi, Hamzah Malik, Nicola C. J. E. Eraut, Merribeth J. Morin, Laura J. Szewczyk, George R. Painter. Human Safety, Tolerability, and Pharmacokinetics of a Novel Broad-Spectrum Oral Antiviral Compound, Molnupiravir, with Activity Against SARS-CoV-2. medrxiv, doi:https://doi.org/10.1101/2020.12.10.20235747 (2020).

8 Good, S. S. et al. AT-527, a Double Prodrug of a Guanosine Nucleotide Analog, Is a Potent Inhibitor of SARS-CoV-2 In Vitro and a Promising Oral Antiviral for Treatment of COVID-19. Antimicrob. Agents Chemother. 65, doi:10.1128/AAC.02479-20 (2021).

9 Beigel, J. H. et al. Remdesivir for the Treatment of Covid-19 - Final Report. N. Engl. J. Med. 383, 1813-1826, doi:10.1056/NEJMoa2007764 (2020).

10 Oude Munnink, B. B. et al. Transmission of SARS-CoV-2 on mink farms between humans and mink and back to humans. Science 371, 172-177, doi:10.1126/science.abe5901 (2021).

11 Richard, M. et al. SARS-CoV-2 is transmitted via contact and via the air between ferrets. Nat Commun 11, 3496, doi:10.1038/s41467-020-17367-2 (2020).

12 Felsenstein, S. \& Hedrich, C. M. SARS-CoV-2 infections in children and young people. Clin. Immunol. 220, 108588, doi:10.1016/j.clim.2020.108588 (2020).

13 Zhu, N. et al. A Novel Coronavirus from Patients with Pneumonia in China, 2019. N. Engl. J. Med. 382, 727-733, doi:10.1056/NEJMoa2001017 (2020).

14 Tracking SARS-CoV-2 variants". www.who.int. Retrieved 1 June 2021. (2021).

15 Faria, N. R. et al. Genomics and epidemiology of the P.1 SARS-CoV-2 lineage in Manaus, Brazil. Science 372, 815-821, doi:10.1126/science.abh2644 (2021). 
16 Humeniuk, R. et al. Pharmacokinetic, Pharmacodynamic, and Drug-Interaction Profile of Remdesivir, a SARS-CoV-2 Replication Inhibitor. Clin. Pharmacokinet. 60, 569-583, doi:10.1007/s40262-021-00984-5 (2021).

17 Fiege, J. K. et al. Single cell resolution of SARS-CoV-2 tropism, antiviral responses, and susceptibility to therapies in primary human airway epithelium. PLoS Pathog. 17, e1009292, doi:10.1371/journal.ppat.1009292 (2021).

18 bei RESOURCES https://www.beiresources.org/Catalog/animalviruses/NR-54982.aspx retrieved on 06/09/2021.miel, A. M. et al. In vitro evolution of Remdesivir resistance reveals genome plasticity of SARS-CoV-2. bioRxiv, 2021.2002.2001.429199, doi:10.1101/2021.02.01.429199 (2021).

20 Agostini, M. L. et al. Coronavirus Susceptibility to the Antiviral Remdesivir (GS-5734) Is Mediated by the Viral Polymerase and the Proofreading Exoribonuclease. mBio 9, doi:10.1128/mBio.00221-18 (2018).

21 Gordon, C. J. et al. Remdesivir is a direct-acting antiviral that inhibits RNA-dependent RNA polymerase from severe acute respiratory syndrome coronavirus 2 with high potency. J. Biol. Chem. 295, 6785-6797, doi:10.1074/jbc.RA120.013679 (2020).

22 Tchesnokov, E. P. et al. Template-dependent inhibition of coronavirus RNA-dependent RNA polymerase by remdesivir reveals a second mechanism of action. J. Biol. Chem. 295, 1615616165, doi:10.1074/jbc.AC120.015720 (2020).

23 Park, S. J. et al. Antiviral Efficacies of FDA-Approved Drugs against SARS-CoV-2 Infection in Ferrets. mBio 11, doi:10.1128/mBio.01114-20 (2020).

24 Shi, J. et al. Susceptibility of ferrets, cats, dogs, and other domesticated animals to SARScoronavirus 2. Science 368, 1016-1020, doi:10.1126/science.abb7015 (2020).

25 Maltezou, H. C. et al. Association Between Upper Respiratory Tract Viral Load, Comorbidities, Disease Severity, and Outcome of Patients With SARS-CoV-2 Infection. J. Infect. Dis. 223, 1132-1138, doi:10.1093/infdis/jiaa804 (2021).

26 de Wit, E. et al. Prophylactic and therapeutic remdesivir (GS-5734) treatment in the rhesus macaque model of MERS-CoV infection. Proc. Natl. Acad. Sci. U. S. A. 117, 6771-6776, doi:10.1073/pnas.1922083117 (2020).

27 Williamson, B. N. et al. Clinical benefit of remdesivir in rhesus macaques infected with SARSCoV-2. Nature 585, 273-276, doi:10.1038/s41586-020-2423-5 (2020).

28 Faria, N. R. et al. Genomics and epidemiology of a novel SARS-CoV-2 lineage in Manaus, Brazil. medRxiv, doi:10.1101/2021.02.26.21252554 (2021).

29 Lo, M. K. et al. Remdesivir targets a structurally analogous region of the Ebola virus and SARSCoV-2 polymerases. Proceedings of the National Academy of Sciences 117, 26946-26954, doi:10.1073/pnas.2012294117 (2020).

30 Kutter, J. S. et al. SARS-CoV and SARS-CoV-2 are transmitted through the air between ferrets over more than one meter distance. Nat Commun 12, 1653, doi:10.1038/s41467-021-21918-6 (2021).

31 Abdool Karim, S. S. \& de Oliveira, T. New SARS-CoV-2 Variants - Clinical, Public Health, and Vaccine Implications. N. Engl. J. Med. 384, 1866-1868, doi:10.1056/NEJMc2100362 (2021).

32 Pearson, C. A. B. et al. Estimates of severity and transmissibility of novel SARS-CoV-2 variant 501Y.V2 in South Africa. (https://cmmid.github.io/topics/covid19/sa-novel-variant.html. . London: CMMID Repository (2021).

33 Davies, N. G. et al. Estimated transmissibility and impact of SARS-CoV-2 lineage B.1.1.7 in England. Science 372, doi:10.1126/science.abg3055 (2021). 
34 Addetia, A. et al. Sensitive Recovery of Complete SARS-CoV-2 Genomes from Clinical Samples by Use of Swift Biosciences' SARS-CoV-2 Multiplex Amplicon Sequencing Panel. J. Clin. Microbiol. 59, doi:10.1128/JCM.02226-20 (2020).

35 Greninger, A. L. et al. Rapid Metagenomic Next-Generation Sequencing during an Investigation of Hospital-Acquired Human Parainfluenza Virus 3 Infections. J. Clin. Microbiol. 55, 177-182, doi:10.1128/JCM.01881-16 (2017).

36 Bolger, A. M., Lohse, M. \& Usadel, B. Trimmomatic: a flexible trimmer for Illumina sequence data. Bioinformatics 30, 2114-2120, doi:10.1093/bioinformatics/btu170 (2014).

37 Lin, M. J., Shean, R. C., Makhsous, N. \& Greninger, A. L. LAVA: a streamlined visualization tool for longitudinal analysis of viral alleles. bioRxiv, 2019.2012.2017.879320, doi:10.1101/2019.12.17.879320 (2019).

$38 \mathrm{Li}, \mathrm{H}$. \& Durbin, R. Fast and accurate short read alignment with Burrows-Wheeler transform. Bioinformatics 25, 1754-1760, doi:10.1093/bioinformatics/btp324 (2009).

39 Koboldt, D. C. et al. VarScan: variant detection in massively parallel sequencing of individual and pooled samples. Bioinformatics 25, 2283-2285, doi:10.1093/bioinformatics/btp373 (2009).

40 Koboldt, D. C., Larson, D. E., Chen, K., Ding, L. \& Wilson, R. K. Massively parallel sequencing approaches for characterization of structural variation. Methods Mol. Biol. 838, 369-384, doi:10.1007/978-1-61779-507-7_18 (2012).

41 Wang, K., Li, M. \& Hakonarson, H. ANNOVAR: functional annotation of genetic variants from high-throughput sequencing data. Nucleic Acids Res. 38, e164, doi:10.1093/nar/gkq603 (2010). 
a

352

353

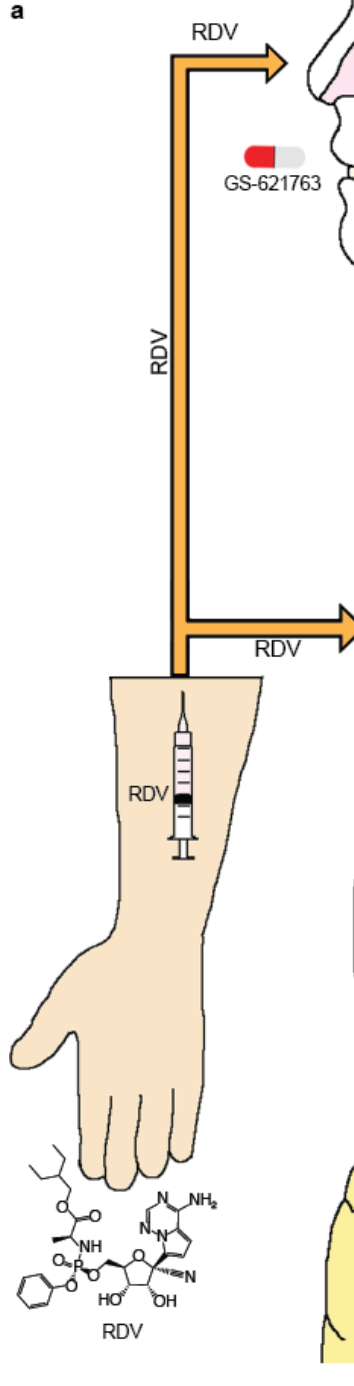

RDV
GS-441524

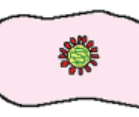

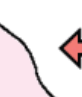
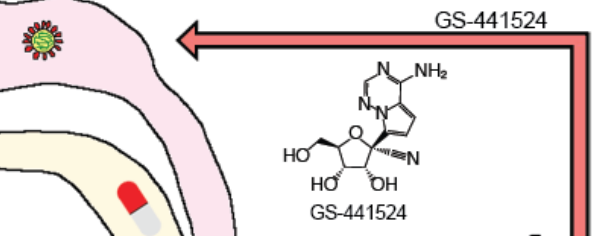

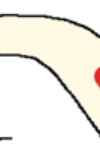
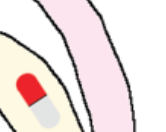

Ho

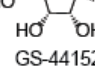

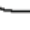

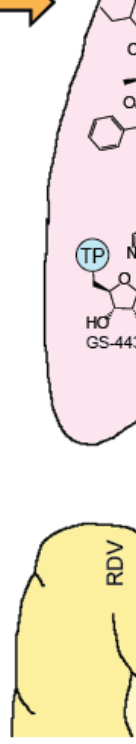

b

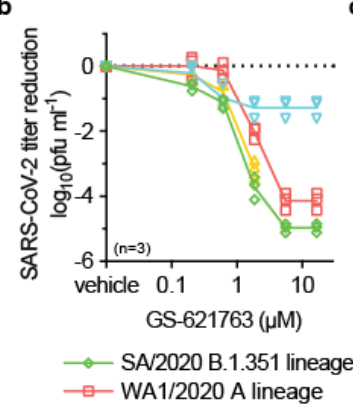

c

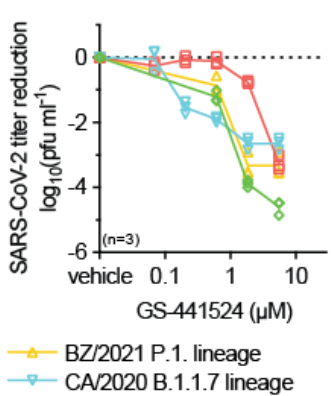

d
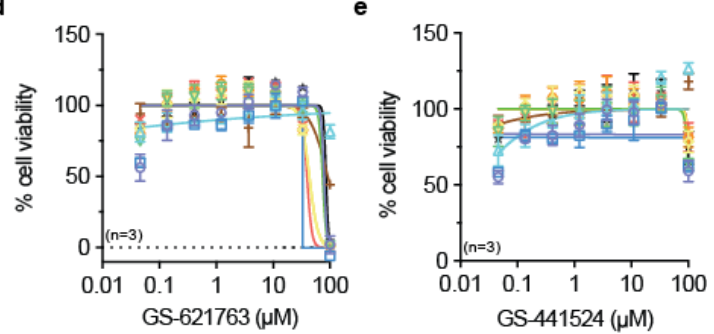

"F2" HAE —— "M2" HAE

"F3" HAE — "DF2" HAE

Fig. 1. Antiviral potency of remdesivir analog GS-621763. a, Schematic depicting metabolism of remdesivir (RDV) and GS-621763 after injection or oral uptake, respectively. Remdesivir distributes into tissues (e.g. lung) and is efficiently metabolized intracellularly to GS-443902. Conversely, GS441524 is the dominant plasma metabolite after intestinal absorption of orally administered GS-627163 and is subsequently anabolized to GS-443902 in the tissues. b-c, Virus yield reduction of SARS-CoV-2 clinical isolates WA1/2020, CA/2020, SA/2020, and BZ/2021 representing the A, B.1.1.7 ( $\alpha$ ), B.1.351 $(\beta)$ and P.1 $(\gamma)$ lineages, respectively, by GS-621763 (b) and GS-441524 (c) on VeroE6 cells. EC 50 concentrations are specified. d-e, In vitro cytotoxicity profiles of GS-621763 (d) and GS-441524 (e) on 
361 VeroE6, HEp-2, BHK-21, HCT-8 and a panel of primary HAE cells from independent donors ("F2", 362 "F3", "M2", "M6", "DF2"). In (b-e), symbols represent individual biological repeats (n=3), error bars 363 show standard deviations, lines depict non-linear regression models.

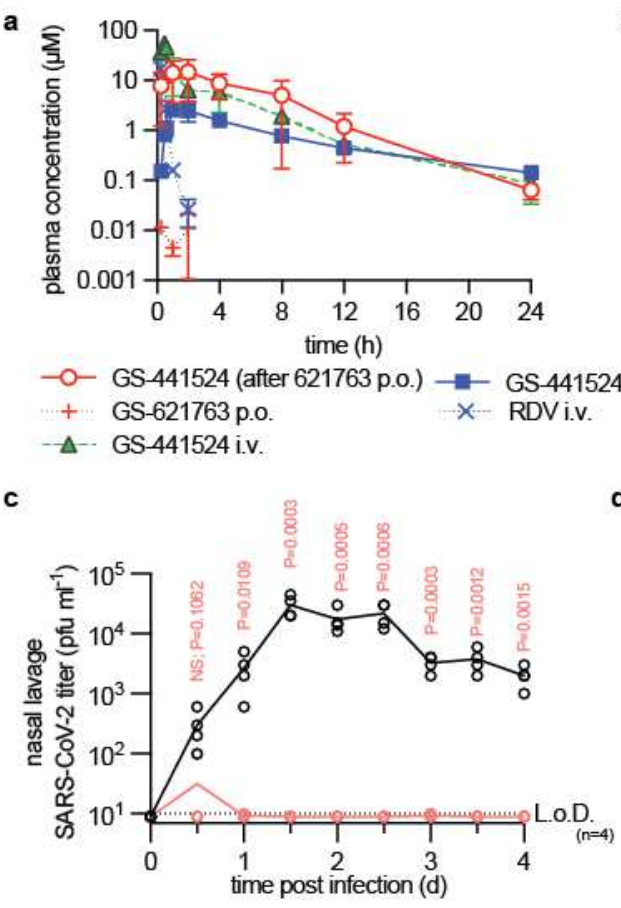

b

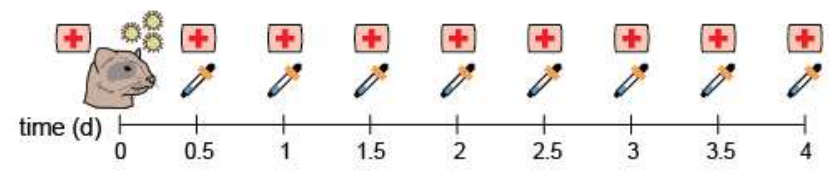

SARS-CoV-2 $1 \times 10^{5}$ p.f.u. nasal lavage

\# GS-621763 [20 mg/kg; b.i.d.; 0 h.p.i.]

$$
\text { (after RDV i.v.) }
$$

d
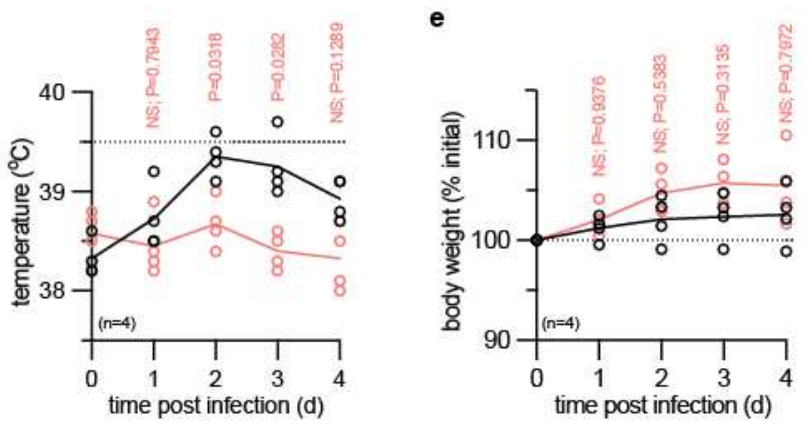

$\rightarrow$ vehicle $\quad \longrightarrow$ GS-621763 [20 $\mathrm{mg} \mathrm{kg}^{-1}$ b.i.d. $]$

f

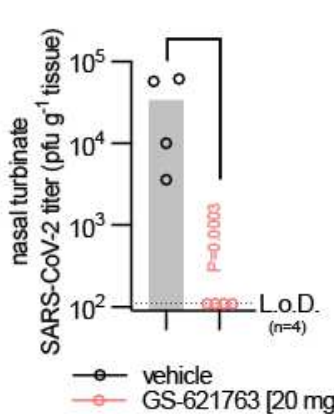

g

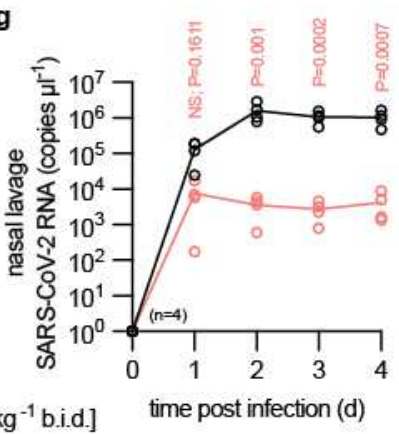

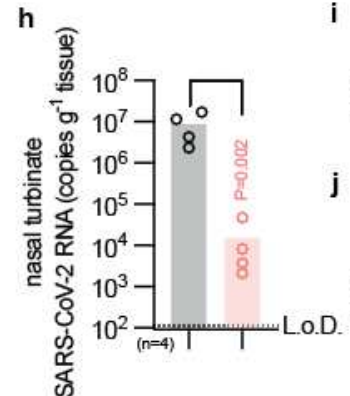

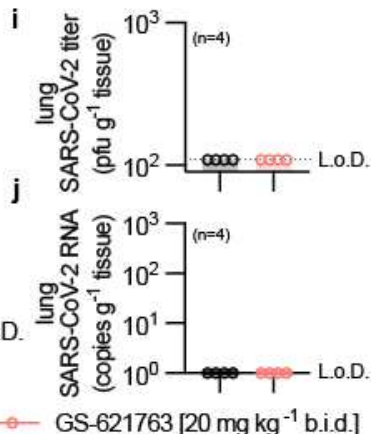

366

367

368

369

Fig. 2. Prophylactic efficacy of GS-621763. a, Single-dose PK study in ferrets showing plasma concentrations of GS-441524, GS-621763, and remdesivir (RDV) as specified after dosing with GS621763 (30 mg/kg; p.o.), remdesivir (10 mg/kg; i.v.), and GS-441524 (20 mg/kg; i.v.). Symbols represent individual biological repeats $(n=3)$, error bars show standard deviations, lines depict sample 
370 means. b, Schematic of the prophylactic efficacy study design. Ferrets were infected intranasally with $3711 \times 10^{5}$ pfu WA1/2020 (virus symbol). Groups $(\mathrm{n}=4)$ were gavaged b.i.d. (first aid symbol) with vehicle 372 or GS-621763 (20 mg/kg) starting at the time of infection. Nasal lavages (pipet symbol) were harvested 373 twice daily. All animals were terminated 4 days after infection. c, Virus titers from nasal lavages; LoD, 374 limit of detection. d, Temperature measurements collected once daily. e, Body weight measured once 375 daily. f, Infectious titers of SARS-CoV-2 in nasal turbinates harvested four days after infection. $\mathbf{g}$, 376 SARS-CoV-2 RNA copies present in nasal lavages. $\mathbf{h}$, SARS-CoV-2 RNA copies detected in nasal 377 turbinates. i-j, SARS-CoV-2 infectious particles (i) and SARS-CoV-2 RNA copies (j) in lungs four days 378 after infection. The number of independent biological repeats (individual animals) is shown in each 379 subpanel, symbols represent independent biological repeats, lines (c, d, e, g) and bar graphs (f, h-j) 380 connect or show samples mean, respectively, and P values are stated. 2-way ANOVA with Sidak's post381 hoc multiple comparison tests (c, d, e, g) or two tailed t-test (f, h). 


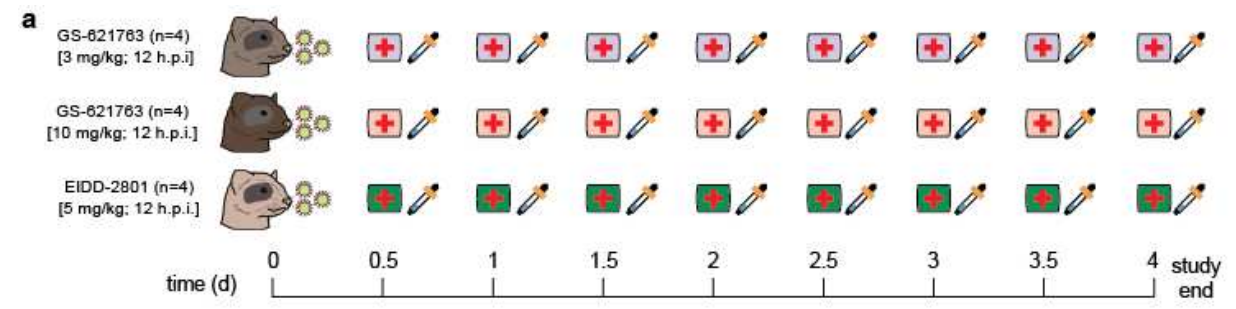

SARS-CoV-2 $1 \times 10^{5}$ pfu

- nasal lavage

¥ GS-621763 [3 mg/kg; b.i.d.; 12 h.p.i.]

$¥$ GS-621763 [10 mg/kg; b.i.d.; 12 h.p.i.]

EIDD-2801 [5 mg/kg; b.i.d.; 12 h.p.i.]
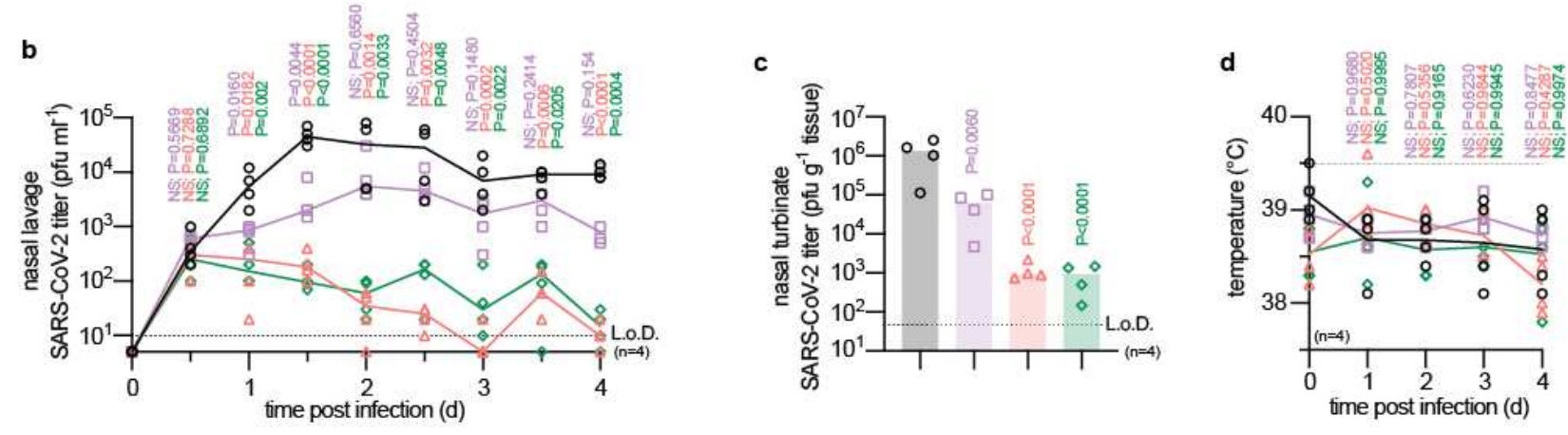

$\rightarrow$ vehicle

$\because$ GS-621763 [3 mg/kg; b.i.d.]

GS-621763 [10 mg/kg; b.i.d]

EIDD-2801 [5 mg/kg; b.id.]

e

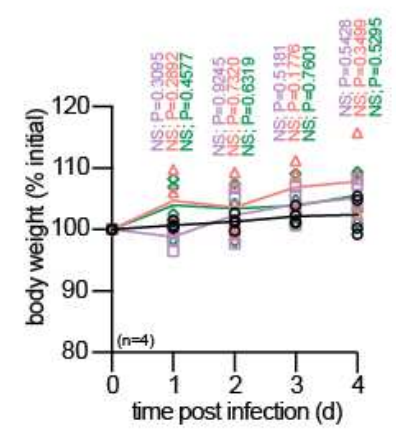

f

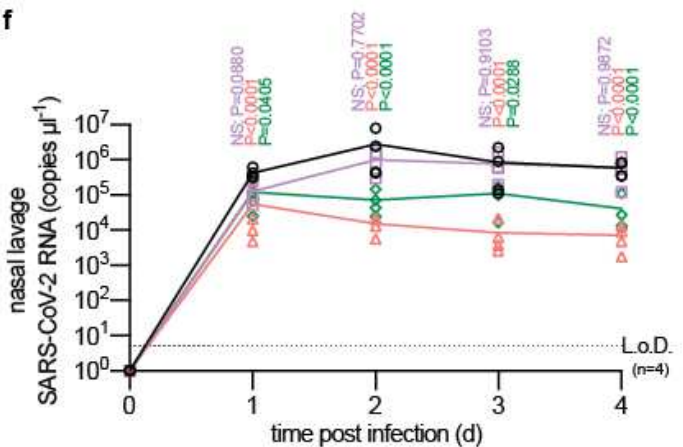

g

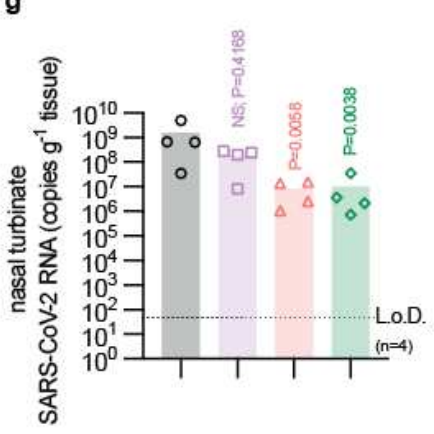

$\rightarrow$ vehicle $\quad-$ GS-621763 [3 mg/kg; b.i.d] $\rightarrow$ GS-621763 [10 mg/kg; b.i.d.] $\rightarrow$ EIDD-2801 [5 mg/kg; b.i.d]

383 Fig. 3. Therapeutic efficacy of GS-621763. a, Schematic of the therapeutic efficacy study design.

384 Ferrets were infected intranasally with $1 \times 10^{5} \mathrm{pfu}$ WA1/2020. Symbols as described for Fig. 2a. Starting 38512 hours after infection, groups of ferrets $(\mathrm{n}=4)$ were gavaged b.i.d. with vehicle GS-621763 (3 mg/kg (purple first aid symbol) or $10 \mathrm{mg} / \mathrm{kg}$ (pink first aid symbol)), or treated with EIDD-2801 (5 mg/kg 387 (green first aid symbol)). Nasal lavages were harvested twice daily. Animals were terminated 4 days 388 after infection. b, Virus titers from nasal lavages. c, Infectious titers of SARS-CoV-2 in nasal turbinates 389 harvested four days after infection. d, Temperature measurements collected once daily. e, Body weight measured once daily. f, SARS-CoV-2 RNA copies present in nasal lavages. g, SARS-CoV-2 RNA 
391 copies detected in nasal turbinates. The number of independent biological repeats (individual animals) is 392 shown in each subpanel. Symbols represent independent biological repeats, lines (b, d, e, f) and bar 393 graphs (c, g) connect or show samples mean, respectively, and P values are stated. 1-way (c, g) or 2-way 394 (b, d, e, f) ANOVA with Dunnett's post-hoc multiple comparison tests. 


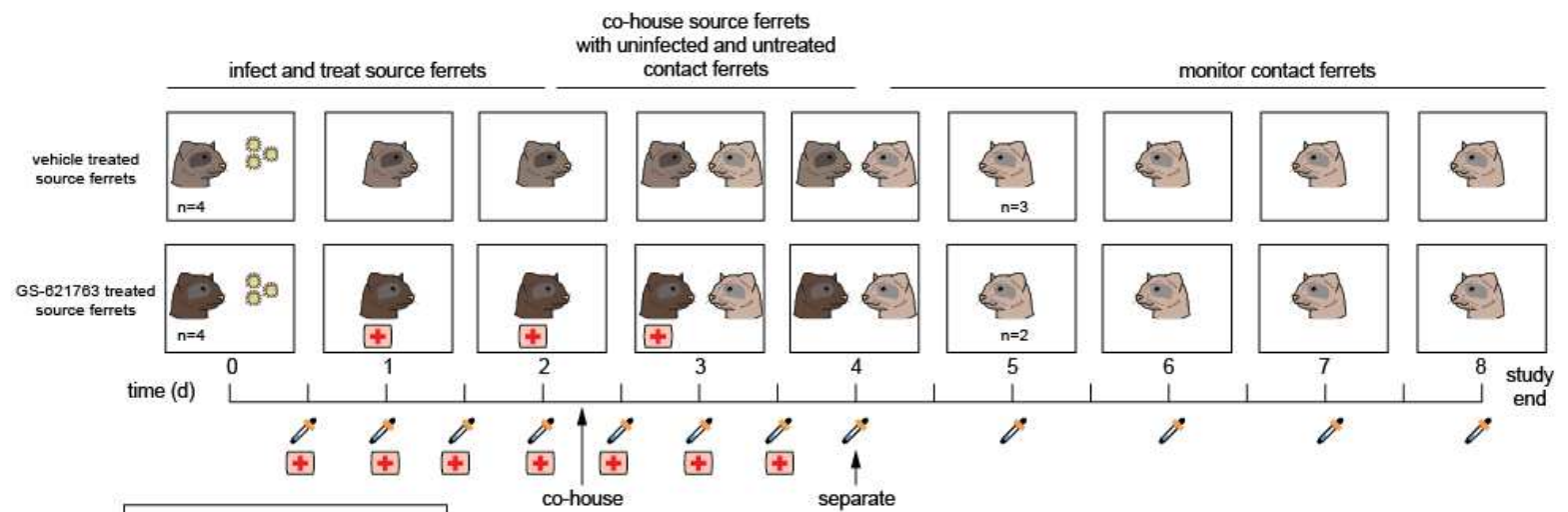

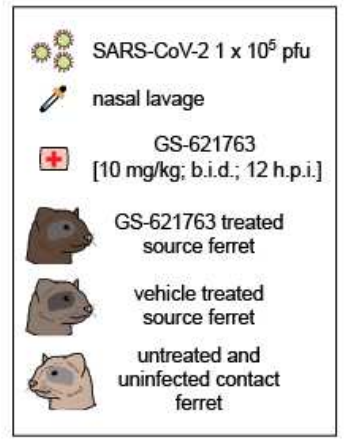

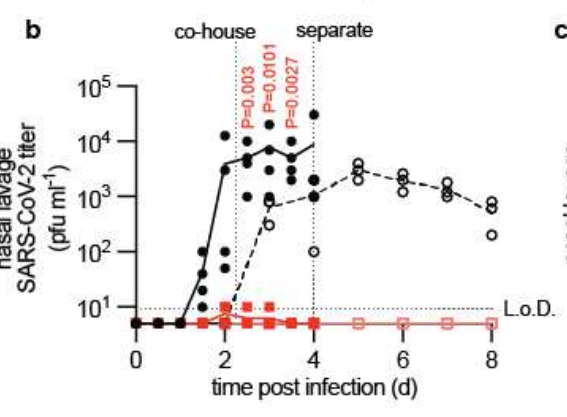

$$
\rightarrow \text { vehicle }(n=4)
$$$$
\rightarrow \text { GS-621763 [10 } \mathrm{mg} / \mathrm{kg} \text {; b.i.d.] }(\mathrm{n}=4)
$$

d

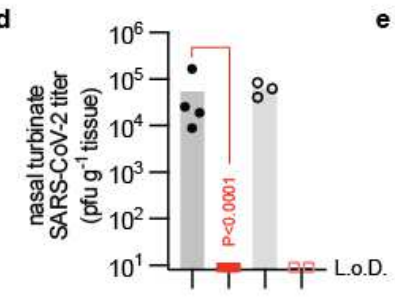

- vehicle $(n=4)$

- GS-621763 [10 mg/kg; b.i.d.] $(\mathrm{n}=4)$

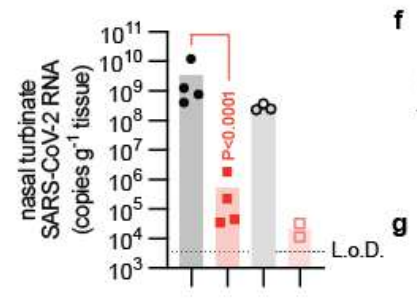

o untreated contact - vehicle $(n=3)$

- untreated contact - GS-621763 ( $n=2)$ c

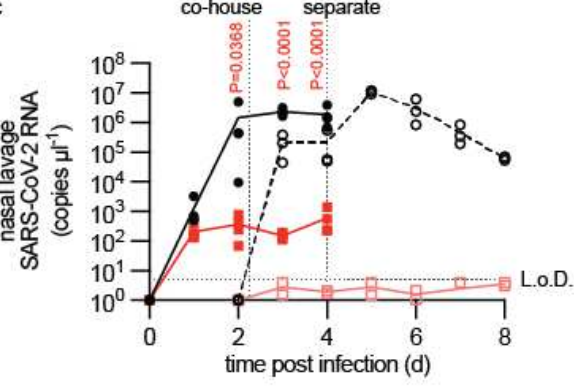

$-\rightarrow-$ untreated contact - vehicle $(n=3)$

untreated contact - GS-621763 $(n=2)$
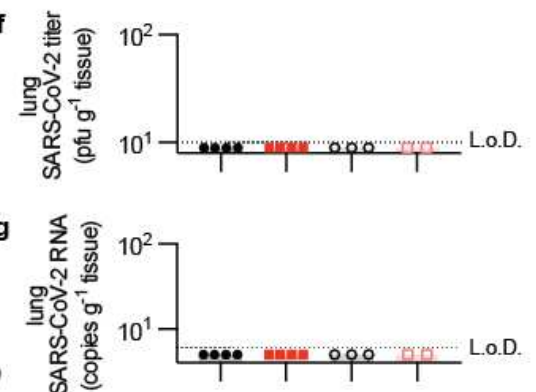

h

BZ/2021 WA/2020

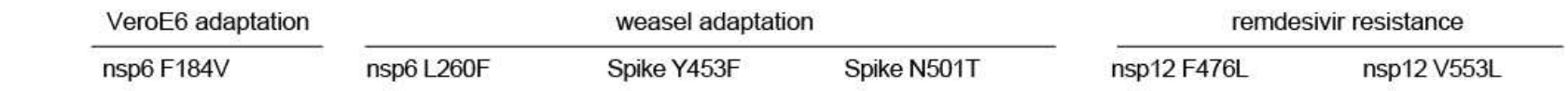
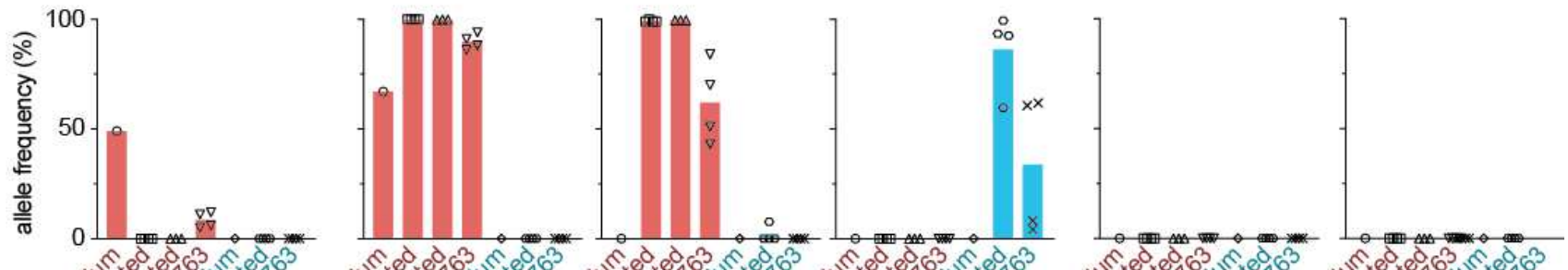
Fig. 4. GS-621763 blocks replication and transmission of SARS-CoV-2 VoC BZ/2021. a, Schematic of the efficacy and contact transmission study design. All ferrets were infected intranasally with $1 \times 10^{5}$ pfu BZ/2021 (virus symbol). Symbols as described for Fig. 2a. Groups of ferrets $(n=4)$ were gavaged b.i.d. with vehicle or GS-621763 (10 mg/kg) starting at 12 hours after infection. Nasal lavages were harvested twice daily. Source ferrets were terminated 4 days after infection and all contact animals were 401 terminated on study day 8. b, Virus titers from nasal lavages. c, SARS-CoV-2 RNA copies present in 402 nasal lavages. d, Infectious titers of SARS-CoV-2 in nasal turbinates harvested four days after infection. e, SARS-CoV-2 RNA copies detected in nasal turbinates. f, Infectious titers of SARS-CoV-2 in lung tissue. g, SARS-CoV-2 RNA copies present in lung tissue. In (b-g), the number of independent biological repeats (individual animals) is shown in each subpanel. Symbols represent independent 406 biological repeats, lines $(\mathrm{b}, \mathrm{c})$ and bar graphs $(\mathrm{d}, \mathrm{e}, \mathrm{f}, \mathrm{g})$ connect or show sample means, respectively, and $\mathrm{P}$ values are stated. 1-way $(\mathrm{d}, \mathrm{e})$ or 2-way $(\mathrm{b}, \mathrm{c})$ ANOVA with Tukey’s $(\mathrm{d}, \mathrm{e})$ or Sidak's $(\mathrm{b}, \mathrm{c})$ post-

408 hoc multiple comparison tests. $\mathbf{h}$, Metagenome sequence analysis of inoculum WA1/2020 and BZ/2021 409 viruses, virus populations extracted from ferret nasal turbinates four days after infection, and BZ/2021 410 populations extracted from nasal lavages of contacts of vehicle-treated source animals. Relative allele 411 frequencies of signature residues are shown. Symbols represent independent biological repeats (virus 412 population of individual animals), columns show group means.

\section{Methods}

\section{Study design}

Ferrets (Mustela putorius furo) were used as an in vivo model to determine efficacy of the orally 417 available remdesivir analog GS-621763 against SARS-CoV-2 infection and transmission to untreated 418 and uninfected contact animals. Oral bioavailability and pharmacokinetic properties of GS-621763 in 
419 ferrets were assessed prior to infecting any animals with SARS-CoV-2. For efficacy and transmission 420 studies, anesthetized ferrets were inoculated intranasally with SARS-CoV-2. Nasal lavages were

421 performed periodically at predefined time points to measure virus load. Temperature and body weight 422 were measured once daily for all animals. At four days after infection (study day 8 for contact ferrets), 423 animals were euthanized and nasal turbinates extracted to measure virus load in the upper respiratory 424 tract. SARS-CoV-2 titers were determined by plaque assay and viral RNA copies quantified by RT425 qPCR.

\section{Cells and viruses}

African green monkey kidney VeroE6 (ATCC®, cat\# CRL-1586 ${ }^{\mathrm{TM}}$ ), human lung adenocarcinoma epithelial Calu-3 (ATCC® HTB-55 ${ }^{\mathrm{TM}}$ ), human epithelial/HeLa contaminant HEp-2 (ATCC®, cat\# CCL-23 ${ }^{\mathrm{TM}}$ ), and baby hamster kidney BHK-21 (ATCC ${ }^{\text {, }}$, cat\# CCL-10 ${ }^{\mathrm{TM}}$ ) cells were cultivated in a 431 cat\# 10-013-CV, lot\# 05721000) supplemented with 7.5\% (10\% for Calu-3) heat-inactivated fetal 432 bovine serum (FBS) (Corning, cat\# 35-010-CV, lot\# 14020001). Human epithelial colon 433 adenocarcinoma HCT-8 cells (ATCC® cat\# CCL-244 ${ }^{\mathrm{TM}}$ lot $\# 70036111$ ) were cultivated at $37^{\circ} \mathrm{C}$ and $5 \%$ $434 \mathrm{CO}_{2}$ in Roswell Park Memorial Institute (RPMI) medium (Quality biological, cat\# 112-024-101, lot\# 435 723411) supplemented with $2 \mathrm{mM}$ L-glutamine (Gibco, cat\# 23030-081) and 10\% heat-inactivated FBS. A549-hACE2 cells that stably express human angiotensin-converting enzyme 2 (hACE2) were 437 grown in the culture medium supplemented with $10 \mu \mathrm{g} / \mathrm{mL}$ Blasticidin S. Primary human airway 438 epithelial (HAE) cells from multiple donors were cultivated at $37^{\circ} \mathrm{C}$ and $5 \% \mathrm{CO}_{2}$ in Bronchial Epithelial 439 Cell Growth Medium (BEGM) BulletKit following the provider's instructions (Lonza, cat\# CC-3171 440 lot\# 0000889952 with supplement cat\# CC-4175 lot\# 0000848033). Human Bronchial Tracheal 441 Epithelial cells (HBTEC) were derived from the following donors: "F2" from a 29-year old Caucasian 
442 female (Lifeline, cat\# FC-0035, lot\# 5101); "F3" from a 42-year old Caucasian female (Lonza, cat\# CC443 2540S, lot\# 0000519670); "M2" from a 40-year old Caucasian male (Lonza, cat\# CC-2540S, lot\# 444 0000667744); and "M6" from a 48-year old Caucasian male (Lonza, cat\# CC-2540S, lot\# 0000544414). 445 Diseased (Asthma) Human Bronchial Epithelial (DHBE) cells "DF2" were from a 55-year old 446 Caucasian female (Lonza, cat\# 00194911S, lot\# 0000534647). Primary HAE were used for cytotoxicity 447 assays at passage $\leq 3$. Cell lines were routinely checked for mycoplasma and bacterial contamination. 448 SARS-CoV-2 strains were obtained from BEI and propagated using Calu-3 cells supplemented with $4492 \%$ FBS in accordance with approved biosafety level 3 protocols. Virus stocks were stored at $-80^{\circ} \mathrm{C}$.

450 Stock virus titers were determined by plaque assay and stocks authenticated through metagenomic 451 sequencing.

452 Plaque assays

453 Vero E6 cells were seeded in 12-well plates at $3 \times 10^{5}$ cells per well. The following day, samples 454 were serially diluted in DMEM containing Antibiotic-Antimycotic (Gibco) supplemented with 2\% FBS. 455 Dilutions were then added to cells and incubated for 1 hour at $37^{\circ} \mathrm{C}$. Cells were subsequently overlayed 456 with 1.2\% Avicel 581-NF (FMC BioPolymer) in DMEM containing Antibiotic-Antimycotic (Gibco) 457 and allowed to incubate for 3 days at $37^{\circ} \mathrm{C}$ with $5 \% \mathrm{CO}_{2}$. After 3 days, the overlay was removed, cells 458 were washed once with phosphate buffered saline (PBS) and fixed with neutral buffered formalin (10\%) 459 for 15 minutes. Plaques were then visualized using $1 \%$ crystal violet followed by washing with water.

460 Compound sources and chemical synthesis

461 Remdesivir was either purchased from MedChemExpress (cat\# HY-104077, batch\# 46182) or 462 synthesized at Gilead Sciences, Inc. GS-441524 was either purchased from MedChemExpress (cat\# HY463 103586, batch\# 62110) or synthesized at Gilead Sciences Inc. GS-621763 was synthesized at Gilead 
464 Sciences Inc. All EIDD-2801/molnupiravir used in this study was provided by Gilead Sciences Inc., 465 sourced from MedChemExpress.

\section{Cytotoxicity assays}

467 7,500 cells were seeded in each well of 96-well plates (Corning, cat\# 3598). Cells were incubated 468 with 3-fold serial dilutions of compound from a $100 \mu \mathrm{M}$ maximum concentration. Each plate included 4 469 wells of positive (100 $\mu \mathrm{M}$ cycloheximide (Millipore Sigma, cat\# C7698-5G)) and negative (vehicle $470(0.2 \%$ dimethyl sulfoxide (DMSO) $)$ controls for normalization. Plates were incubated in a humidified 471 chamber at $37^{\circ} \mathrm{C}$ and $5 \% \mathrm{CO}_{2}$ for 72 hours. PrestoBlue ${ }^{\mathrm{TM}}$ Cell Viability Reagent (ThermoFisher 472 Scientific, cat\# A13262) was added in each well (10 $\mu 1 /$ well) and fluorescence recorded on a Synergy 473 H1 multimode microplate reader (BioTek) after 1-hour incubation (excitation $560 \mathrm{~nm}$, emission 590 $474 \mathrm{~nm})$. Raw data was normalized with the formula: $\%$ cell viability $=100 \times($ signal sample - signal positive 475 control) / ( signal negative control - signal positive control). 50\% cytotoxic concentrations $\left(\mathrm{CC}_{50}\right)$ and 95

$476 \%$ confidence intervals after non-linear regression were determined using the inhibitor vs normalized 477 response equation in Prism 9.1.0 for MacOS (GraphPad). For cytotoxicity assays in A549-hACE2 cells, 478 compounds $(200 \mathrm{~nL})$ were spotted onto 384-well plates prior to seeding 5000 A549-hACE2 cells/well in 479 a volume of $40 \mu \mathrm{L}$ culture medium. The plates were incubated at $37^{\circ} \mathrm{C}$ for 48 hours with $5 \% \mathrm{CO}_{2}$. On 480 day 2, $40 \mu \mathrm{L}$ of CellTiter-Glo (Promega) was added and mixed 5 times. Plates were read for 481 luminescence on an Envision (PerkinElmer) and $\mathrm{CC}_{50}$ values calculated using a nonlinear four 482 parameter regression model.

483 Virus yield reduction

$4842 \times 10^{5}$ VeroE6 cells were seeded per well in 12-well plates 16 hours before infection. Confluent 485 monolayers were then infected with the indicated virus at a multiplicity of infection (MOI) of 0.1 $486 \mathrm{pfu} /$ cell for 1 hour at $37^{\circ} \mathrm{C}$ with frequent rocking. Inoculum was removed and replaced with $1 \mathrm{~mL}$ of 
DMEM with $2 \%$ FBS and the indicated concentration of compound. Cells were incubated at $37^{\circ} \mathrm{C}$ and $5 \% \mathrm{CO}_{2}$ for 48 hours. Supernatant were harvested, aliquoted and stored at $-80^{\circ} \mathrm{C}$ before being analyzed 489 by plaque assay.

\section{Reporter virus assays}

A549-hACE2 cells (12,000 cells per well in medium containing 2\% FBS) were plated into a white clear-bottomed 96-well plate (Corning) at a volume of $50 \mu$ l. On the next day, compounds were added 493 directly to cultures as 3-fold serial dilutions with a Tecan D300e digital liquid dispenser, with DMSO 494 volumes normalized to that of the highest compound concentration (final DMSO concentration $<0.1 \%$ ). 495 The diluted compound solutions were mixed with $50 \mu \mathrm{l}$ of SARS-CoV-2-Nluc (MOI 0.025 pfu/cell), 496 expressing a nano luciferase reporter protein (kind gift of Xuping Xie and Pei-Yong Shi (University of 497 Texas Medical Branch; Galveston, TX), were added. At 48 h post-infection, $75 \mu$ l Nano luciferase 498 substrate solution (Promega) was added to each well. Luciferase signals were measured using an 499 Envision microplate reader (Perkin Elmer). The relative luciferase signals were calculated by 500 normalizing the luciferase signals of the compound-treated groups to that of the DMSO-treated groups 501 (set as $100 \%) . \mathrm{EC}_{50}$ values were calculated using a nonlinear four parameter variable slope regression 502 model.

\section{Pharmacokinetics}

504 Female ferrets were either intravenously administered $10 \mathrm{mg} / \mathrm{kg}$ remdesivir as a 30-minute infusion or 505 orally administered $30 \mathrm{mg} / \mathrm{kg} \mathrm{GS}-621763$, after which plasma was isolated at 7 to 9 timepoints post 506 administration. Plasma samples underwent methanol protein precipitation followed by centrifugation. 507 Resulting supernatants were isolated, evaporated to dryness under nitrogen and reconstituted with 5\% 508 acetonitrile for injection onto an LC-MS/MS system. Concentrations of remdesivir, GS-621763 and GS509441524 were determined using 9-point calibration curves spanning at least 3 orders of magnitude, with 
510 quality control samples to ensure accuracy and precision, prepared in normal ferret plasma. Analytes

511 were separated by a $50 \times 3.0 \mathrm{~mm}, 2.55 \mu \mathrm{m}$ Synergi Polar-RP 30A column (Phenomenex, Inc.) using a

512 mobile phase A consisting of $10 \mathrm{mM}$ ammonium formate with $0.1 \%$ formic acid and a mobile phase B

513 consisting of $0.1 \%$ formic acid in acetonitrile. A multi-stage linear gradient from 5\% to $95 \%$ mobile

514 phase $\mathrm{B}$ at a flow rate of $1 \mathrm{~mL} / \mathrm{min}$ was employed for analyte separation. Pharmacokinetic parameters

515 were calculated using Phoenix WinNonlin (version 8.2, Certara) and concentration-time profiles

516 generated using Prism (version 8, GraphPad).

\section{$517 \quad$ Ferret efficacy studies}

518 Female ferrets (6-10 months old, Mustela putorius furo) were purchased from Triple F Farms.

519 Ferrets were rested for 7 days after arrival. Ferrets were then housed individually or in groups of 2 in

520 ventilated negative-pressure cages in an ABSL-3 facility. Based on previous experiments ${ }^{6}$, ferrets were

521 randomly assigned to groups $(\mathrm{n}=4)$ and used as an in vivo model to examine the efficacy of orally

522 administered compounds against SARS-CoV-2 infection. No blinding of investigators was performed.

523 Ferrets were anesthetized using dexmedetomidine/ketamine and infected intranasally with $1 \times 10^{5} \mathrm{pfu}$

524 2019-nCoV/USA-WA1/2020 in $1 \mathrm{~mL}$ (0.5 mL per nare). Body weight and temperature were measured

525 once daily. Nasal lavages were performed twice daily using $1 \mathrm{~mL}$ sterile PBS (containing Antibiotic-

526 Antimycotic (Gibco)). Nasal lavage samples were stored at $-80^{\circ} \mathrm{C}$ until virus titration could be

527 performed by plaque assay. Treatment (once daily (q.d.) or twice daily (b.i.d.)) was initiated at either 0

528 or 12 hours after infection and continued until 4 days post infection with either vehicle $(2.5 \%$ dimethyl

529 sulfoxide; 10\% Kolliphor HS-15; 10\% Labrasol; 2.5\% propylene glycol; 75\% water) or compound. Four

530 days after infection, ferrets were euthanized, and tissues and organs were harvested and stored at $-80^{\circ} \mathrm{C}$

531 until processed.

532 Contact transmission in ferrets 
Eight ferrets were anesthetized and inoculated intranasally with $1 \times 10^{5} \mathrm{pfu}$ of hCoV-19/Japan/TY7-

$534503 / 2021$. Twelve hours after infection, ferrets were split into two groups ( $\mathrm{n}=4 ; 2$ ferrets per cage) and 535 treated with vehicle or GS-621763 $\left(10 \mathrm{mg} \mathrm{kg}^{-1}\right)$ twice daily (b.i.d.) via oral gavage. At 54 hours after 536 infection, uninfected and untreated contact ferrets (two contacts for GS-621763; three contacts for 537 vehicle) were co-housed with source ferrets. Co-housing was continued until 96 hours after infection 538 and source ferrets were euthanized. Contact ferrets were housed individually and monitored for an 539 additional 4 days after separation from source ferrets and subsequently euthanized. Nasal lavages were 540 performed on all source ferrets every 12 hours and all contact ferrets every 24 hours. For all ferrets, 541 nasal turbinates and lung tissues were harvested to determine viral titers and the detection of viral RNA.

\section{SARS-CoV-2 titration in tissue extracts}

543 Selected tissues were weighed and mechanically homogenized in sterile PBS. Homogenates were 544 clarified by centrifugation $(2,000 \times \mathrm{g})$ for 5 minutes at $4^{\circ} \mathrm{C}$. Clarified homogenates were then serially 545 diluted and used in plaque assays to determine virus titer as described above.

\section{Quantitation of SARS-CoV-2 RNA copy numbers}

To probe viral RNA in selected tissues, samples were harvested and stored in RNAlater at $-80^{\circ} \mathrm{C}$.

Total RNA from tissues was isolated using a RNeasy mini kit (Qiagen), in accordance with the manufacturer's protocol. For nasal lavage samples, total RNA was extracted using a ZR viral RNA kit (Zymo Research) in accordance with the manufacturer's protocol. SARS-CoV-2 RNA was detected as 551 previously described ${ }^{6}$ using the $\mathrm{nCoV}$ IP2 primer-probe set (National Reference Center for Respiratory 552 Viruses, Pasteur Institute). An Applied Biosystems 7500 using the StepOnePlus real-time PCR system 553 was used to perform $\mathrm{RT}$-qPCR reactions. The $\mathrm{nCoV}$ IP2 primer-probe set was using in combination 554 with TaqMan fast virus 1-step master mix (Thermo Fisher Scientific) to detect viral RNA. SARS-CoV-2 555 RNA copy numbers were calculated using a standard curve created from serial dilutions of a PCR 
556 fragment (12669-14146 nt of the SARS-CoV-2 genome), as previously described ${ }^{6}$. For RNA copies in

557 tissue samples, RNA copies were normalized to the weights of the tissues used.

\section{Next generation sequencing}

559 To authenticate virus stocks, metagenomic sequencing was performed as described ${ }^{34,35}$, while to 560 sequence lower viral load in vivo samples the COVID-Seq (Illumina) amplicon tiling protocol was used. 561 For metagenomic sequencing, viral RNA was treated with Turbo DNase I (Thermo Fisher), converted to 562 cDNA using random hexamers and SuperScript IV reverse transcriptase, and double stranded cDNA 563 created using Sequenase v2.0. Sequencing libraries for both sets of libraries were generated using 564 Nextera Flex (illumina) and cleaned using $0.8 \times$ Ampure XP beads and pooled equimolarly before 565 sequencing on an Illumina $1 \times 100$ bp NextSeq2000 run. Raw fastq reads were adapter- and quality566 trimmed with Trimmomatic v0.39 ${ }^{36}$. To interrogate potential resistance alleles, reference-based 567 mapping to NC_045512.2 was carried out using our modified Longitudinal Analysis of Viral Alleles 568 (LAVA - https://github.com/michellejlin/lava) ${ }^{37}$ pipeline. LAVA constructs a candidate reference 569 genome from early passage virus using $\mathrm{bwa}^{38}$, removes PCR duplicates with Picard, calls variants with 570 VarScan ${ }^{39,40}$, and converts these changes into amino acid changes with Annovar ${ }^{41}$. Consensus sequences 571 were called with TAYLOR ${ }^{34}$ and deposited in NCBI GenBank. Accession numbers (supplementary 572 Table 4) are as follows: input strain WA1/2020, MZ433205; WA1/2020 recovered from ferrets, 573 MZ433206 - MZ433213; input strain BZ/2021, MZ433225; BZ/2021 recovered from source ferrets, 574 MZ433214 - MZ433221; BZ/2021 recovered from contacts of vehicle-treated source ferrets, MZ433222 575 - MZ433224. Raw reads for these sequences are publicly available on SRA (BioProject PRJNA740065). 576 Ethics statement

All in vivo efficacy studies were conducted at Georgia State University in compliance with the 578 Animal Welfare Act Code of Federal Regulations and the Guide for the Care and Use of Laboratory 
579 Animals of the National Institutes of Health. All studies involving SARS-CoV-2 infected ferrets were

580 approved by the Georgia State Institutional Animal Care and Use Committee under protocol A20031.

581 Experiments at Georgia State University using infectious SARS-CoV-2 were performed in BSL-

582 3/ABSL-3 facilities at Georgia State University and approved by the Georgia State Institutional

583 Biosafety Committee under protocol B20016. Experiments at Gilead Sciences, Inc. using infectious

584 SARS-CoV-2 were performed in a BSL-3 facility and approved by an institutional biosafety committee.

585 Statistics and reproducibility

586 The Microsoft Excel (version 16.48), GraphPad Prism (version 9.1.0), and Numbers (version 10.1)

587 software packages were used for data collection and analysis. One-way or two-way ANOVA with

588 Dunnett's or Tukey's multiple comparisons post-hoc test were used to evaluate statistical significance

589 when comparing more than two groups or two independent variables. When comparing two variables, a

590 two-tailed unpaired t-test was performed to determine statistical significance. The specific statistical test

591 used to individual studies is specified in the figure legends. RT-qPCR data were collected and analyzed

592 using the StepOnePlus (version 2.1; Applied Biosystems) software package. Final figures were

593 assembled in Adobe Illustrator (version CS6). All numerical raw data and summaries of individual

594 statistical analyses are provided in supplementary datasets 3 and 4 . Effect sizes between groups in the

595 ANOVAs were calculated as $\eta^{2}=\left(\mathrm{SS}_{\text {effect }}\right) /\left(\mathrm{SS}_{\text {total }}\right)$ for one-way ANOVA and $\omega^{2}=\left(\mathrm{SS}_{\text {effect }}-\right.$

$\left.596\left(\mathrm{df}_{\text {effect }}\right)\left(\mathrm{MS}_{\text {error }}\right)\right) / \mathrm{MS}_{\text {error }}+\mathrm{SS}_{\text {total }}$ for two-way ANOVA $\left(\mathrm{SS}_{\text {effect, }}\right.$ sum of squares for the effect; $\mathrm{SS}_{\text {total, }}$

597 sum of squares for total; $\mathrm{df}_{\text {effect, }}$, degrees of freedom for the effect; $\mathrm{MS}_{\text {error }}$, mean squared error). The

598 statistical significance level $\alpha$ was set to $<0.05$ for all experiments. Exact $\mathrm{P}$ values are shown in the

599 individual graphs.

600

601 Acknowledgements 
We thank BEI Resources for SARS-CoV-2 isolates, X. Xie and P.-Y. Shi for providing SARS-

603 CoV-2-Nluc, A. Shornikov of the Gilead Sample Bank group for compound distribution, the High

604 Containment Core and the Department for Animal Research of Georgia State University for support, and 605 A. L. Hammond and D. Porter for critical reading of the manuscript.

606

607

\section{Author Contributions}

J. P. B. and R.K.P. coordinated the study. R.M.C. and J.S. performed virus-stock preparations and

609 virus titrations. R.M.C., J.D.W., C.M.L., and R.K.P. performed animal inoculations, nasal lavage 610 sampling, animal necropsies, and/or titration of virus from ferrets R.M.C. extracted RNA from all

611 animal samples and performed all RT-qPCR experiments and analyses. J.S. performed virus reduction 612 assays and cytotoxicity assessments. D.B. coordinated pharmacokinetics studies in ferrets. K.B. 613 prepared compound formulations. V.D.P., J.P.B., and J.C. performed reporter virus and cytotoxicity 614 assays in ACE2-A549 cells. R.K, K.C, and R.L.M. synthesized and sourced compounds. M.J.L. and 615 A.L.G. performed all next-generation sequencing and analyses. R.M.C. created all figure schematics. 616 R.M.C., J.S., J.P.B., and R.K.P. were responsible for experimental design, data analysis, and data 617 presentation. R.M.C., J.D.W., C.M.L., J.S., D.B., L.S.M., A.L.G., R.L.M., J.P.B., and T.C. edited the 618 manuscript. J.P.B. and R.K.P. conceived and designed the study. R.K.P. wrote the manuscript.

Funding

This work was supported by Gilead Sciences Inc. and, in part, by Public Health Service grants 622 AI153400 (to RKP) and AI141222 (to RKP) from the NIH/NIAID. NIH/NIAID had no role in study 623 design, data collection and interpretation, or the decision to submit the work for publication. 


\section{Competing Interests Declaration}

626 All authors affiliated with Gilead Sciences may hold stock or stock options in Gilead Sciences Inc. 627 R.K.P. was the principal investigator of a Gilead-sponsored research agreement with Georgia State 628 University. He has received funding from Gilead Sciences Inc. to support parts of this work. All other 629 authors declare no competing interests.

630

631 Additional Information

632 Supplementary Information

633 Supplementary Information is available for this paper.

635 Correspondence

636 Correspondence and requests for materials should be addressed to Richard K Plemper.

\section{Data Availability}

All data generated and analyzed during this study are included in this published article (and its 640 supplementary information files). Source data and statistical analyses for figures 1-4, extended data 641 tables 1-3 and extended data figures 1-2 are provided with the paper in supplementary datasets 3-4. 642

\section{Code Availability}

644 This study does not use proprietary codes. All computer codes and algorithms used are specified in 645 the Methods section. 
Extended Data Table 1. Antiviral potency and cytotoxicity.

\begin{tabular}{|c|c|c|c|c|c|c|c|}
\hline \multirow[b]{2}{*}{ virus } & \multirow[b]{2}{*}{ host cells } & \multicolumn{2}{|c|}{ GS-621763 } & \multicolumn{2}{|c|}{ GS-441524 } & \multicolumn{2}{|c|}{ remdesivir } \\
\hline & & $\begin{array}{l}\mathrm{EC}_{50} \\
{[\mu \mathrm{M}]}\end{array}$ & $\begin{array}{l}\mathrm{CC}_{50} \\
{[\mu \mathrm{M}]}\end{array}$ & $\mathrm{EC}_{50}[\mu \mathrm{M}]$ & $\begin{array}{l}\mathrm{CC}_{50} \\
{[\mu \mathrm{M}]}\end{array}$ & $\mathrm{EC}_{50}[\mu \mathrm{M}]$ & $\mathrm{CC}_{50}[\mu \mathrm{M}]$ \\
\hline $\begin{array}{l}\text { WA1/2020-nano } \\
\text { luciferase }\end{array}$ & A549-ACE2 & $0.98^{a}$ & $>50^{\mathrm{b}}$ & $1.4 \pm 0.9^{c}$ & $>50^{\mathrm{b}}$ & $\begin{array}{c}0.075 \pm \\
0.03^{\mathrm{d}} \\
\end{array}$ & $17.3 \pm 4.9^{e}$ \\
\hline WA1/2020f & VeroE6 & 0.73 & $>100$ & 0.68 & $>100$ & n.d. & $>100$ \\
\hline $\mathrm{CA} / 2020(\alpha)^{f}$ & VeroE6 & 0.21 & $>100$ & 0.11 & $>100$ & n.d. & $>100$ \\
\hline SA/2020 $(\beta)^{\dagger}$ & VeroE6 & 0.11 & $>100$ & 0.34 & $>100$ & n.d. & $>100$ \\
\hline BZ/2021 $(\gamma)^{f}$ & VeroE6 & 0.22 & $>100$ & 0.55 & $>100$ & n.d. & $>100$ \\
\hline n.a. ${ }^{f}$ & HEp-2 & n.a. & 79.4 & n.a. & $>100$ & n.a. & 45.12 \\
\hline n.a. ${ }^{f}$ & VeroE6 & n.a. & $>100$ & n.a. & $>100$ & n.a. & $>100$ \\
\hline n.a. ${ }^{f}$ & BHK-21 & n.a. & $>100$ & n.a. & $>100$ & n.a. & $>100$ \\
\hline n.a.f & HCT-8 & n.a. & 74.6 & n.a. & $>100$ & n.a. & 36.4 \\
\hline n.a. ${ }^{f}$ & "F2" HAE & n.a. & 43.8 & n.a. & $>100$ & n.a. & 85.5 \\
\hline n.a. ${ }^{f}$ & "F3" HAE & n.a. & 43.8 & n.a. & $>100$ & n.a. & 104.4 \\
\hline n.a. ${ }^{f}$ & "M2" HAE & n.a. & 39.7 & n.a. & $>100$ & n.a. & 101.9 \\
\hline n.a. ${ }^{f}$ & “M6" HAE & n.a. & 92.3 & n.a. & $>100$ & n.a. & $>100$ \\
\hline n.a. ${ }^{f}$ & "DF2" HAE & n.a. & 86 & n.a. & $>100$ & n.a. & $>33$ \\
\hline
\end{tabular}

$650{ }^{a}$ mean $(n=2)$; data represent mean of two independent experiments, each with technical duplicates.

$651{ }^{b}$ mean $(n=2)$; data represent mean of two independent experiments, each with technical triplicates or 652 quadruplicates.

$653{ }^{c}$ mean $\pm \mathrm{SD}(\mathrm{n}=8)$; data represent mean of eight independent experiments, each with technical 654 duplicates.

$655{ }^{d}$ mean $\pm \mathrm{SD}(\mathrm{n}=12)$; data represent mean of twelve independent experiments, each with technical 656 duplicates.

657 emean $\pm \mathrm{SD}(\mathrm{n}=6)$; data represent mean of six independent experiments, each with technical triplicates 658 or quadruplicates.

659 mean $(n=3)$; all other data represent mean of three independent experiments. 


\begin{tabular}{|c|c|c|c|c|c|c|c|}
\hline compound & route & $\begin{array}{c}\text { dose } \\
{[\mathrm{mg} / \mathrm{kg}]}\end{array}$ & $\begin{array}{c}\mathbf{t}_{1 / 2} \\
{[\text { hours] }}\end{array}$ & $\begin{array}{c}\text { IV - CL [L/hours/kg] } \\
\text { PO - T } \\
\max \\
{[\text { hours }]}\end{array}$ & $\begin{array}{l}\mathrm{C}_{\max } \\
{[\mu \mathrm{M}]}\end{array}$ & $\begin{array}{l}\text { AUC } \mathbf{C}_{\text {last }} \\
{[\boldsymbol{\mu M} \mathbf{M} . \mathbf{h}]}\end{array}$ & $\begin{array}{c}\mathbf{F} \\
{[\%]}\end{array}$ \\
\hline GS-441524 & i.v. & 20 & 3.4 & 0.86 & 54.2 & 81.1 & $\mathrm{n} / \mathrm{a}$ \\
\hline remdesivir & i.v. & 10 & 6.09 & $\mathrm{n} / \mathrm{a}$ & 2.81 & 18.2 & $\mathrm{n} / \mathrm{a}$ \\
\hline GS-621763 ${ }^{\mathrm{a}}$ & p.o. & 30 & $2.68 \pm 0.15$ & $4.0 \pm 3.5$ & $15.8 \pm 4.7$ & $80.8 \pm 14.6$ & $\begin{array}{c}115 \pm \\
21\end{array}$ \\
\hline
\end{tabular}

663 aapproximately $10 \mathrm{nM}$ GS-621763 transiently observed in first two hours

664

665 Extended Data Table 3. GS-441524 and its metabolite concentrations in ferret lung tissue.

\begin{tabular}{c|c|c|c|c} 
compound & route & dose & $\begin{array}{c}\text { lung GS-443902 } \\
{[\mathbf{n m o l} / \mathbf{g}]}\end{array}$ & $\begin{array}{c}\text { lung total nuc } \\
{[\mathbf{n m o l} / \mathbf{g}]}\end{array}$ \\
\hline GS-441524 & i.v. & $20 \mathrm{mg} \mathrm{kg}^{-1}$ & $0.53 \pm 0.10$ & $0.66 \pm 0.21$ \\
\hline remdesivir & i.v. & $10 \mathrm{mg} \mathrm{kg}^{-1}$ & $1.28^{\mathrm{a}}$ & 2.96 \\
\hline GS-621763 & p.o. & $30 \mathrm{mg} \mathrm{kg}^{-1}$ & $0.30 \pm 0.19$ & $0.88 \pm 0.13$
\end{tabular}

${ }^{\mathrm{a}}$ one lung from remdesivir i.v. dosing was BLQ for all metabolites

666

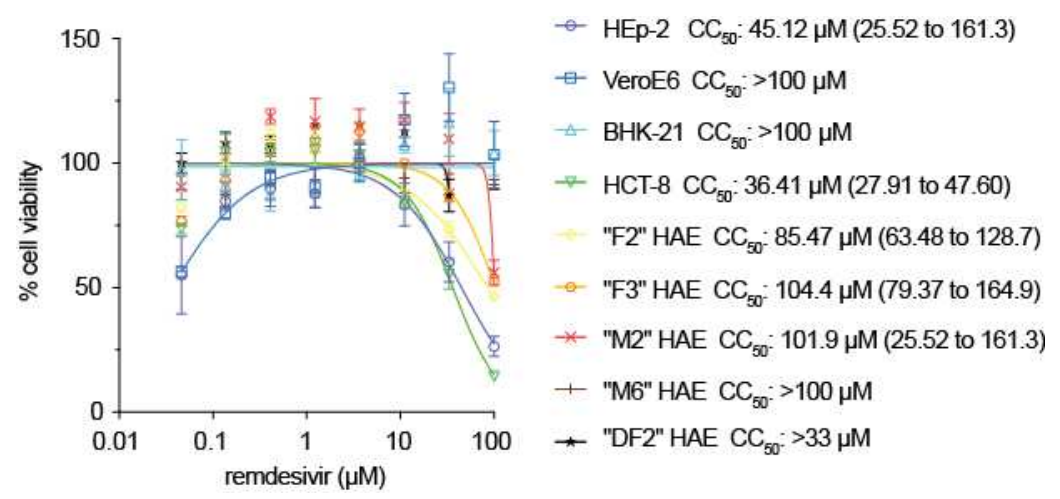

668 Extended Data Fig. 1. Remdesivir cytotoxicity. In vitro cytotoxicity profile of remdesivir on HEp-2,

669 VeroE6, BHK-21, HCT-8 and the panel of primary HAE cells (“F2”, "F3”, "M2”, "M6", "DF2”) cells

670 examined in (Fig. 2b-e). Symbols represent individual biological repeats $(n=3)$, error bars show standard

671 deviations, lines depict non-linear regression models.

672 


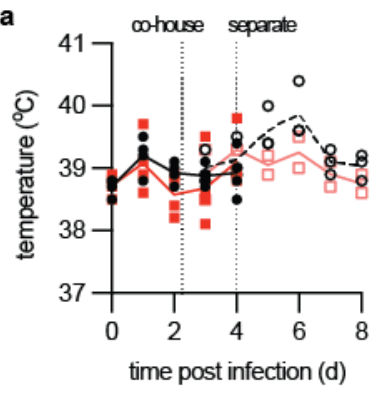

b

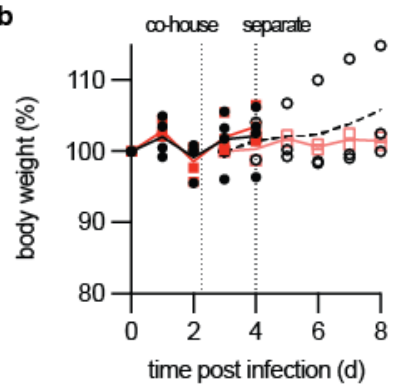

673

$\rightarrow \quad$ vehicle $(\mathrm{n}=4)$

- $\odot-. \quad$ untreated contact - vehicle $(n=3)$

untreated contact - GS-621763 $(n=2)$

674 Extended Data Fig. 2. Clinical signs in source and contact animals infected with BZ/2021. a,

675 Temperature measurements collected once daily. b, Body weight measured once daily. 


\section{Supplementary Files}

This is a list of supplementary files associated with this preprint. Click to download.

- SupplementaryInformation.pdf

- Supplementarydataset1.html

- Supplementarydataset2.html 